weist. Der Lagenunterschied der Minima für die beiden Polarisationsrichtungen würde dann daher rühren, daß die bei der Reflexion auftretenden Phasensprünge im Totalreflexionsgebiet für die beiden Schwingungskomponenten verschieden groß sind. Um dies zu sichern, stellte sich als lohnend heraus, einmal den gesamten Verlauf der Phasensprünge von senkrechter bis zu streifender Inzidenz in einem Schaubild zusammenzufassen. (Man erkennt an dieser Darstellung, die gesondert mitgeteilt werden soll, zugleich die Quelle der Widersprüche, die sich über eine so bekannte Erscheinung sogar zwischen verschiedenen klassischen Darstellungen der Optik finden.) Für die vorliegende Erscheinung zeigt sich, daß die Parallelkomponente jeweils das innere, die senkrechte das äußere Glied des Paars bil- den sollte. Die Beobachtung zeigt die entgegengesetzte Lage (Abb.3). Da auch die Absolutwerte der Aufspaltung um ein Mehrfaches größer sind, als der Elliptizität entspräche, ist zu schließen, daß Aufdampfschichten - auch aus von Natur aus nicht doppelbrechendem Material häufig doppelbrechend sind.

Zur Erläuterung der Brechzahlbestimmung dient u. a. eine Aufnahmereihe an Kryolith $(n=1,364)$ (Abb.4), die je nach dem Druck des beim Aufdampfen anwesenden Fremdgases Brechzahlen der Schicht von 1,05 an aufwärts ergibt. Die Dichte der beim höchsten Fremddruck entstehenden Schichten ist demnach nur ein Zehntel der des kompakten Materials. Der lose Aufbau gibt die Möglichkeit von Ordnungsdoppelbrechung und Streuwirkungen.

\title{
Beobachtungen über Thalliumdiffusion in kristallinem hexagonalem Selen ${ }^{1}$
}

\author{
Von B. Gudden ( $\dagger$ ) und Kurt Lehovec
}

(Z. Naturforschg. 1, 508-511 [1946]; eingegangen am 29. Juni 1946)

\begin{abstract}
In der Nähe des Selen-Schmelzpunktes diffundiert Thallium in kristallinem hexagonalem Selen mit beträchtlicher Geschwindigkeit. Es wandern Thallium-Ionen. Die Diffusion kann spektroskopisch, bequemer jedoch durch die Beeinflussung der Selen-Leitfähigkeit verfolgt werden. Bisher wurden keine anderen Metalle mit ähnlichem Verhalten gefunden.
\end{abstract}

1.

$\mathrm{G}$ elegentlich anderer Untersuchungen wurde ein Diffusionsvorgang beobachtet, der in mancher Hinsicht mit der von R. W. $\mathrm{Pohl}^{2}$ und Mitarbeitern untersuchten Diffusion in Alkalihalogeniden übereinstimmt, im übrigen aber doch ein bisher unbekannter Typ zu sein scheint. Wir berichten über unsere bisherigen Erfahrungen und hoffen $\mathrm{zu}$ gegebener Zeit die Vorgänge genauer erklären zu können.

Untersuchungsgegenstand sind zylindrische Stäbchen von einigen cm Länge und $6 \mathrm{~mm}$ Drchm. aus kristallinem hexagonalem Selen. Die Stäbchen sind aus amorphem Selen (Selen reinst in Perlen von Schering-Kahlbaum) durch mehrstündige Erwärmung auf $216^{\circ} \mathrm{C}$ (zur Vermei-

1 Die Arbeit ist im Institut für experimentelle Physik der Universität Prag entstanden und wurde im Jahr 1944 R. W. Pohl zum 60. Geburtstag überreicht.

${ }_{2}$ Physik. Z. 39, 36 [1938]. dung von Hohlraumbildung unter Druck) entstanden. Die „Leitfähigkeit“"3 solcher Selenproben liegt bei $5 \cdot 10^{-4} \mathrm{Ohm}^{-1} \mathrm{~cm}^{-1}$. Wird auf eine Stirnseite einer derartigen Probe Thallium aufgedampft und diese anschließend einige Zeit auf höherer Temperatur gehalten, so -wandert Thallium ein.

Die Einwanderung läßt sich spektroskopisch verfolgen. $\mathrm{Zu}$ diesem $\mathrm{Zwecke}$ wurden die einseitig mit Thallium bedampften Probestäbchen verschiedene Zeit lang bei $216^{\circ} \mathrm{C}$ im Vakuum gehalten (Vakuum, um Oxydation des Thalliums $\mathrm{zu}$ vermeiden). Sodann wurden in verschiedenen Abständen von der mit Thallium bedampften Stirnfläche Selenproben entnommen und ihr Thalliumgehalt spektroskopisch quantitativ bestimmt.

3 Die Angabe einer „Leitfähigkeit“ am Selen ist eine mißliche Angelegenheit, da der Widerstand vielfach von Spannung, Stromdauer und vor allem der Frequenz des elektrischen Feldes abhängt. Die Zahlen sollen daher nur als Anhalt dienen. 
Es zeigte sich, daß das Thallium mit einer Geschwindigkeit von rund $2 \mathrm{~mm}$ je Tag vorrückt. Die spektroskopische Nachweisgrenze lag bei rd. $10^{-6} \mathrm{~g}$ Tl auf $1 \mathrm{~g}$ Selen.

Da die Eindringtiefe des Thalliums in dieser Anordnung nicht proportional der Quadratwurzel aus der Zeit, sondern eher proportional der Zeit selbst wächst, hat es keinen Sinn, einen Diffusionskoeffizienten anzugeben. Die Diffusion scheint konzentrationsabhängig $\mathrm{zu}$ sein. Untersucht man nämlich die Thalliumeinwanderung aus thalliumhaltigem Selen in Reinselen, so ist das Ficksche Diffusionsgesetz um so genauer erfüllt, je geringer die Thalliumkonzentration gewählt wird. Bei einem Thalliumgehalt des Selens von $3 \%$ ergab sich ein Diffusionskoeffizient $5 \cdot 10^{-8} \mathrm{~cm}^{2} / \mathrm{sec}^{4}$. Ein derartiger Wert ist für Festkörper weder besonders groß noch klein; er liegt immerhin an der oberen Grenze der Diffusion in Metallen (zum Vergleich: $\mathrm{Au}$ in $\mathrm{Pb}$ bei $250^{\circ} \mathrm{C}$ ca. $\left.3,2 \cdot 10^{-7} \mathrm{~cm}^{2} / \mathrm{sec}\right)$, bleibt aber um $3-4$ Größenordnungen hinter den im $\mathrm{Poh}$ lschen Institut untersuchten Diffusionen zurück.

Es war uns überraschend, daß kein anderes von uns daraufhin untersuchtes 'Metall $(\mathrm{Al}, \mathrm{Pb}, \mathrm{Cd}$, $\mathrm{Au}, \mathrm{In}, \mathrm{Cu}, \mathrm{Mg}, \mathrm{Ag}, \mathrm{Te}, \mathrm{Bi}, \mathrm{Zn}, \mathrm{Sn}$ ) vergleichbare Diffusion im Selen zeigt; ein Sonderverhalten von $\mathrm{Hg}$ in bezug auf Se ist bekannt, aber nicht näher geklärt. Wir schließen daraus, daß bestimmte chemische Vorgänge maßgeblich beteiligt sind.

Wegen der Langwierigkeit der spektroskopischen Messungen suchten wir nach einem bequemeren Nachweisverfahren. Optische Beobachtung schied aus, wenn wir von einer Beobachtung absehen, die am Schluß dieses Kapitels besprochen wird. Ein bequemes Nachweisverfahren ergab sich jedoch in der Verfolgung der Widerstandsänderung; es ist freilich, wie wir begründen werden, nur mit großer Zurückhaltung zu verwenden.

Legt man an ein einseitig mit $\mathrm{Tl}$ bedampftes Selenstäbchen eine Spannung - Größenordnung 100 Volt - und bestimmt elektrometrisch die Potentialverteilung, so ergibt sie sich streng linear; der „spezifische Widerstand“ ist also im Stäbchen räumlich konstant. Wiederholt man die Messung nach Wärmebehandlung bei $216^{\circ} \mathrm{C}$ und kühlt nachher nicht allzu rasch (in einigen Min.)

${ }^{4}$ Gemessen wurde nicht spektroskopisch, sondern, wie unten angegeben wird, der Potentialverlauf. auf Raumtemperatur ab, so ist der Gesamtwiderstand um mehrere Zehnerpotenzen gestiegen. Die Potentialverteilung ist jetzt nicht im geringsten mehr linear; es ist auch keineswegs der Widerstand in der spektroskopisch $\mathrm{zu}$ bestimmenden Thallium-Einwanderungszone als ganzer erhöht oder gesenkt, sondern eine extreme Widerstandserhöhung ist gerade etwa dort eingetreten, wo die Thalliumeinwanderung die spektroskopische Nachweisgrenze $\left(\mathbf{1 0}^{-6}\right)$ unterschreitet. In den der Aufdampfschicht näheren Bereichen mit höherem Tl-Gehalt ist der spezifische Widerstand zwar ebenfalls gegenüber dem Reinselen erhöht, aber wesentlich kleiner als in entfernteren Bereichen kleineren Tl-Gehaltes (vergl. Abb. 1).

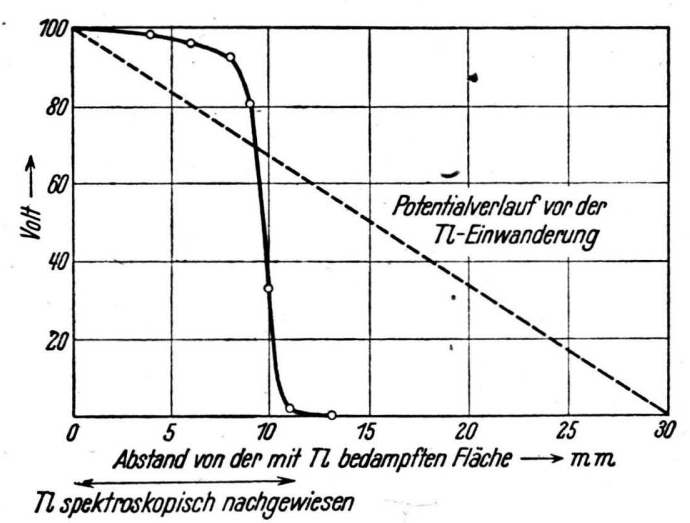

Abb. 1. Potentialverlauf bei Raumtemperatur längs eines Selen-Stäbchens mit einseitiger Thallium-Einwanderung $\left(4 \mathrm{mg} / \mathrm{cm}^{2} \mathrm{Tl}\right.$ auf eine Endfläche aufgedampft und 5 Tage bei $216^{\circ} \mathrm{C}$ im Vakuum gelagert).

Man kann der gemessenen Potentialverteilung entnehmen, daß der spezifische Widerstand im Tl-haltigen Teil mindestens um zwei Zehnerpotenzen höher ist als im "Reinselen“, mit abnehmendem Tl-Gehalt noch um weitere 3 Zehnerpotenzen bis etwa $2 \cdot 10^{8} \mathrm{Ohm} \cdot \overline{\mathrm{cm}}$ steigt, und erst dort, wo der spektroskopische Nachweis versagt (Tl-Konzentration $10^{-6}$ ), rasch auf den $10^{5}$-mal niedrigeren Wert für das „Reinselen“ herabsinkt.

Die Stelle höchsten Widerstandes - stärkster Potentialänderung - läßt sich nun bequem beobachten und aus ihrer Verlagerung auf die Diffusionsvorgänge schließen. Wir haben daher die zeitraubenden spektroskopischen Messungen durch Beobachtung des Potentialverlaufes ersetzt.

Da die lichtelektrische Wirkung dort am größten ist, wo die elektrische Feldstärke am höch- 
sten ist, konnten wir auch mit einer Lichtsonde (Wechsellicht und Verstärker) bequem die Grenze feststellen, bis zu der das Thallium jeweils vorgedrungen war.

\section{2.}

Die Thalliumwanderung (bei $216^{\circ} \mathrm{C}$ ) wird durch ein elektrisches Feld beeinflußt (Abb.2).

Auf die Stirnflächen eines Selenstäbchens wurde beiderseits Thallium aufgedampft. Nach 2 Tagen Wärmebehandlung bei $216^{\circ} \mathrm{C}$ - ohne elektrisches Feld - ergab sich die in Kurve 1 (Abb.2) an-

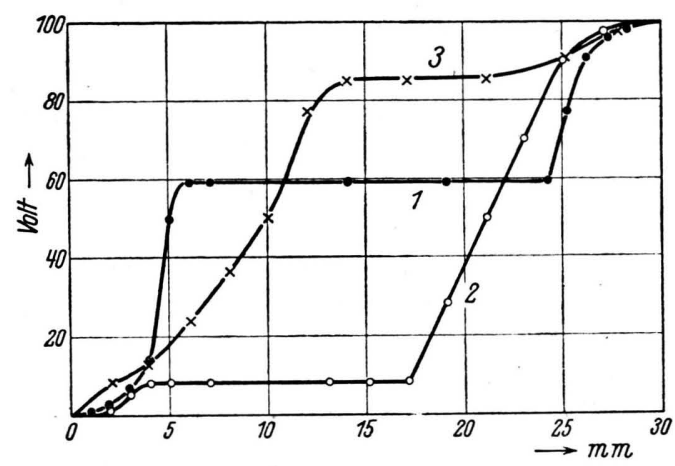

Abb. 2. Beeinflussung des Potentialverlaufs bei Raumtemperatur durch Wärmebehandlung bei $216^{\circ} \mathrm{C}$ im elektrischen Feld (Tl war auf beiden Endflächen aufgedampft worden).

Kurve 1: nach 2 Tagen Wärmebehandlung ohne elektrisches Feld.

Kurve 2: nach weiteren 1,5 Tagen Wärmebehandlung bei 12 Volt; Kathode links.

Kurve 3: nach weiteren 1,5 Tagen Wärmebehandlung bei 12 Volt; Kathode rechts.

gegebene, ziemlich symmetrische Potentialverteilung; das Thallium war beiderseits etwa $5 \mathrm{~mm}$ weit eingewandert. Anschließend wurde die Probe abermals $1 \frac{1}{2}$ Tage bei $\cdot 216^{\circ} \mathrm{C}$ gehalten; diesmal jedoch unter Anlegung einer Gleichspannung von 12 Volt; dabei floß ein Strom von ca. $9 \mu \mathrm{A}$. Danach - bei Raumtemperatur gemessen - war die Potentialverteilung ausgesprochen unsymmetrisch: die kathodennahe Thalliumgrenze war auf die Kathode zu um rd. $2 \mathrm{~mm}$ zurückverschoben; die anodennahe in der gleichen Richtung um $7 \mathrm{~mm}$ vorgerückt. Umpolen und erneute Wärmebehandlung bei $216^{\circ} \mathrm{C}$ von ebenfalls $1^{1 / 2}$ Tagen Dauer ergab eine Verschiebung der Potentialgrenzen in entgegengesetzter Richtung auf die jetzige

5 Vergl. etwa O. St a s i w, Nachr. Ges. Wiss. Göttingen, Math.-physik. Kl. Nr. 50 [1933]; E. M o ll w o u. W. R os s, ebda. (N.F.) 1, 107 [1934].
Kathode (früher Anode) hin. Ersichtlich ist also der Vorgang weitgehend umkehrbar und hat größte Ahnlichkeit mit der im P o hlschen Institut untersuchten Wanderung der Farbzentren im elektrischen Feld ${ }^{5}$.

Offensichtlich diffundieren positive ThalliumIonen; aus Gründen der Elektroneutralität müssen dann zum Ausgleich Elektronen oder SelenIonen wandern. Letzteres käme auf Wanderung von $\mathrm{Tl}_{2} \mathrm{Se}$ oder dergl. hinaus. Da Selen Ersatzleitung hat ${ }^{\mathbf{6}}$, deren Träger immer noch unbekannt sind, verzichten wir vorläufig auf Mitteilung einer

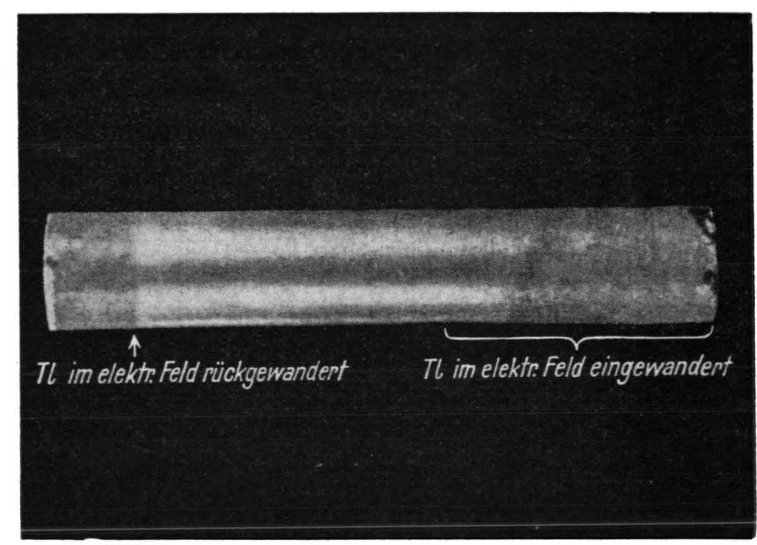

Abb. 3. Zur Thallium-Diffusion in Selen im elektrischen Feld.

genauen Modellvorstellung. Immerhin glauben - wir folgendes sagen zu können: Es handelt sich um ein Indikatorverfahren entsprechend dem im $\mathrm{P}$ o h $\mathrm{l}$ schen Institut benutzten. Das einwandernde Tl macht sich erst infolge einer Reaktion mit einem vorher vorhandenen Bestandteil, der für die elektrische Leitfähigkeit des Selens verantwortlich ist, bemerkbar. Wir denken dabei vor allem an Se-Atome, die nicht im Gitter eingebaut sind. Über die Rolle zusätzlicher Halogenatome hoffen wir später berichten zu können.

\section{3.}

Gelegentlich kann die Grenze der Thalliumeinwanderung mit dem Auge erkannt werden (Abb.3). Dies dürfte nichts mit Ausscheidung einer Thalliumselenidphase oder einer Beeinflussung der optischen Konstanten zu tun haben, sondern be-

${ }^{6}$ F. Eckart u. A. K i t te l, Naturwiss. 29, 371 [1941]. 
ruht wahrscheinlich darauf, daß die Thalliumeinwanderung den Selendampfdruck beeinflußt; dadurch ändert sich die bei $216^{\circ} \mathrm{C}$ schon merkliche Sublimation des Selens, und die geometrische Oberflächenstruktur des Stäbchens erfährt im Bereich der Thalliumeinwanderung eine gewisse Veränderung gegenüber dem im thalliumfreien Teil. Dies macht sich in der diffusen Reflexion bemerkbar.

Abb. 3 zeigt eine scharfe Grenze bei der Rückwanderung und eine verwaschene bei der Einwanderung. Dieser Unterschied ist von der Farbzentrenwanderung in Alkalihalogeniden bekannt und hängt mit der örtlichen Feldstärken-Verteilung zusammen. Aus der Feldstärke-Verteilung bei Raumtemperatur hätte man zunächst im Gegenteil eine scharfe Grenze bei Einwanderung und eine verwaschene Grenze bei Auswanderung erwartet; wir konnten jedoch zeigen, daß Thalliumzusatz bei der Versuchstemperatur von rund $216^{\circ} \mathrm{C}$ keine Widerstandserhöhung wie bei Raumtemperatur, sondern eine Widerstandsverminderung durch zusätzliche $\mathrm{Tl}+++-I o n e n-L e i t-$ fähigkeit bewirkt.

\section{4.}

Die Beeinflussung der elektrischen Eigenschaften von hexagonalem Selen durch Thalliumeinlagerung hängt sehr davon $a b$, mit welcher Geschwindigkeit von der Diffusionstemperatur $\left(216^{\circ} \mathrm{C}\right)$ auf Raumtemperatur abgekühlt wird, der im Bild gezeigte kennzeichnende Potentialverlauf wird nur erhalten, wenn die Abkühlzeit mindestens einige Minuten beträgt. Wird jedoch das Se-Stäbchen in einigen Sekunden abgeschreckt, so ergibt sich ein linearer Potentialverlauf im gesamten Gebiet mit eindiffundiertem $\mathrm{Tl}$.

Offensichtlich hängt die Löslichkeit des Se-Gitters für Tl (vermutlich in irgendeiner Selenidbindung) stark von der Temperatur ab, und bei Abkühlung scheidet sich ein mehr oder weniger hoher Bruchteil als neue Phase aus und wird dadurch elektrisch weitgehend unwirksam. Die Ver-

7 E. M o ll w o, Nachr. Ges. Wiss. Göttingen, Math.physik. Kl. Nr. 25 [1932]; R. V o s e n a c k, Ann. Physik (5) 35, 107 [1939] (bzgl. KJ). hältnisse entsprechen auch hier den im $\mathrm{Pohl-}$ schen Institut beobachteten ${ }^{7}$. Wir müssen annehmen, daß in jedem Falle nur ein sehr kleiner Bruchteil des aufgenommenen Thalliums bei Raumtemperatur molekular (atomar) verteilt ist und als solches Einfluß auf die elektrische Leitung hat; denn der Bereich höchsten Widerstandes fällt mit spektroskopisch bestimmten Thalliumkonzentrationen der Größenordnung um $10^{-6}$ zusammen, während elektrisch selbst für das viel besser leitende Rein-Selen auf Störstellenkonzentration unterhalb $10^{-7}$ geschlossen werden muß.

\section{5.}

Noch ungeklärt ist, ob die Thalliumeinwanderung durch das Selengitter selbst oder nur an den Korngrenzen erfolgt, da wir Kristallite und nicht Einkristalle untersucht haben. Für eine Oberflächenwanderung sprechen vielleicht Messungen im Hochfrequenzfeld, über die wir später berichten werden. Falls es sich aber doch um Wanderungen im Gitter handelt, so bestehen gegenüber den bisher bekannten Diffusionsvorgängen im Festkörper einige wesentliche Unterschiede, die eine genauere Verfolgung unserer Beobachtungen lohnend erscheinen lassen.

Selen hat ein ausgesprochen homöopolares Gitter, bildet also den stärksten Gegensatz zu den heteropolaren wie zu den metallischen Gittern, die bisher ausschließlich auf Diffusionsvorgänge untersucht sind. Die Diffusion des $\mathrm{Tl}$ in diesem verhältnismäßig locker gepackten Gitter erfolgt in Ionenform unter Wirkung spezifisch chemischer Kräfte als stark exothermer Vorgang. Überraschend ist dabei die völlige Sonderstellung des Thalliums gegenüber chemisch nahestehenden Metallen.

Die vorliegende Untersuchung wurde durch den Einfluß von Tl-Spuren auf die Alterung von SelenGleichrichtern angeregt. Hrn. V. J u s h a k o w möchten wir für die Ausarbeitung und Durchführung der spektroskopischen Untersuchungen, Hrn. R. F a u st für die Herstellung der Selen-Proben und der Sü dd e u t s chen A p p a r a e - F a b r i k, Nürnberg, für Bereitstellung der Mittel zur Arbeit danken. 